\title{
Modelling the evolution of galaxies as a function of environment
}

\author{
Gabriella De Lucia
}

\begin{abstract}
In this review, I provide an overview of theoretical aspects related to the evolution of galaxies as a function of environment. I discuss the main physical processes at play, their characteristic time-scales and environmental dependency, and comment on their treatment in the framework of hierarchical galaxy formation models. I briefly summarize recent results and the main open issues.
\end{abstract}

\section{A premise on 'environment'}

Historically, both theoretical and observational studies trying to assess the role of environment on galaxy evolution have been focused on galaxy clusters. One important reason for this is given by the practical advantage of having many galaxies in relatively small regions of the sky, and all approximately at the same distance. As laboratories to study galaxy evolution, however, clusters represent rare and biased systems: they originate from the highest peaks of the primordial density field, and evolutionary processes in these systems are expected to proceed at a somewhat accelerated pace with respect to regions of the Universe with 'average' density. Only about ten per cent of the cosmic galaxy population resides in clusters in the local Universe, and this fraction decreases with increasing redshift.

The recent completion of large spectroscopic and photometric surveys at different cosmic epochs has given new impetus to observational studies trying to assess the role of environment in galaxy evolution. Unfortunately, many different definitions of environment are used in the literature (e.g. some estimate of the halo mass, the number of neighbours counted in some volume, etc), depending on the available observational data, as well as on their quality. This unfortunate but inevitable situation makes results from different surveys and/or at different cosmic epochs difficult to compare, and prevents them from putting really strong constraints on galaxy formation models.

In addition, it should be noted that in order to establish that physical processes related to a particular environment do play a role, it would be necessary to compare the evolution of the same galaxies in different environments. And this is certainly a difficult task from the observational viewpoint. Finally, one should take into account the hierarchical evolution of cosmic structures: dark matter collapses into haloes in a bottom up fashion, with small systems forming first and subsequently merging

INAF - Astronomical Observatory of Trieste, via G. B. Tiepolo 11, I-34143 Trieste, Italy, e-mail: deluciadoats.inaf.it 
to form more massive structures. In this framework, galaxies might experience different 'environments' during their lifetimes. So, for example, galaxies residing in a cluster today might have suffered some degree of 'pre-processing' in lower mass systems like galaxy groups.

\section{Methods}

Hierarchical galaxy formation models find their seeds in the pioneering work by [32]. Galaxies are believed to originate from the condensation of gas at the centre of dark matter haloes: during the collapse of cosmic structure, gas is shock heated to very high temperatures and relaxes to a distribution that exactly parallels that of dark matter. Gas then cools, primarily via thermal Bremsstrahlung, and conservation of angular momentum leads to the formation of a rotationally supported disc. Mergers and instabilities form bulges, that can eventually grow a new disc, provided the system is fed by an appreciable cooling flow.

Different techniques are used to link the observed properties of luminous galaxies to those of the dark matter haloes in which they reside:

- In semi-analytic models of galaxy formation, the evolution of the baryonic components of galaxies is followed using simple yet physically and/or observationally motivated prescriptions to model complex physical processes like star formation, feedback, etc. Modern semi-analytic models take advantage of highresolution N-body simulations to specify the location and evolution of dark matter haloes, which are assumed to represent the birth-places of luminous galaxies. This method can access large dynamic ranges in mass and spatial resolution, and allows a fast exploration of the parameter space, and an efficient investigation of different specific physical assumptions.

- Direct hydrodynamical simulations provide an explicit description of gas dynamics. This method is computationally more expensive then analytic models, so that large cosmological simulations are still somewhat limited by relatively low mass and spatial resolution. In addition, and perhaps most importantly, complex physical processes such as star formation, feedback, etc. still need to be modelled as sub-grid physics, either because the resolution of the simulation becomes inadequate or because (and this is almost always true) we do not have a "complete theory' for the particular physical process under consideration.

- Halo Occupation Distribution (HOD) models are based on a statistical characterization of the link between dark matter haloes and galaxies, bypassing any explicit modelling of the physical processes driving galaxy formation and evolution.

In the following, I will focus primarily on modelling of environmental processes through the first two methods mentioned above (for more details on HOD models, I refer to van den Bosch this volume, and references therein). 


\section{Physical processes}

Theoretically, there are a number of physical processes that can affect the evolution of galaxies in high density environments. In the following, I provide an overview of these processes, and describe how they are included in hierarchical galaxy formation models pointing out the main limitations and problems.

Galaxy mergers : Galaxy mergers and more generally strong galaxy-galaxy interactions, are commonly viewed as a rarity in massive clusters because of the large velocity dispersions of the systems. Mergers are, however, efficient in the infalling group environment, and may represent an important 'preprocessing' step in the evolution of cluster galaxies. Numerical simulations (see e.g. [18, 28, 1, 5] and references therein) have shown that close interactions can lead to a strong internal dynamical response driving the formation of spiral arms and, in some cases, of strong bar modes. Sufficiently close encounters can completely destroy the disc, leaving a kinematically hot remnant with photometric and structural properties that resemble those of elliptical galaxies.

Mergers are intrinsically included in hierarchical galaxy formation models, and represent an important channel for the formation of bulges (see also Wilman, this volume). It should be noted that the number of 'important' mergers increases with increasing stellar mass, but is not as large as commonly thought [8]. In semi-analytic models, galaxy mergers are included adopting some variants of the classical Chandrasekhar dynamical friction formula. The left panel of Fig. 10(from [9]) compares the merger times (in units of dynamical times) adopted in three different semianalytic models with results from numerical simulations by [2]. The figure shows that, over the range of mass-ratios that provide merger times shorter than the Hubble time $\left(M_{\text {sat }} / M_{\text {main }}>0.1\right)$, there are large differences between different models. As discussed in [9], this has important consequences for the assembly history of massive galaxies, in particular of the brightest cluster galaxies (BCGs).

Controlled hydrodynamical simulations of merging disk galaxies are used to 'tune' the efficiency of merger driven starbursts in these models [5]. One important point, often neglected, is that these simulations are usually set-up using structural properties of nearby disk galaxies, which might not necessarily provide a good representation of systems merging at high redshift.

Recent theoretical work ([20] - their Fig. 8 is reproduced in the right panel of Fig. 11 suggests that merges, and more specifically those associated with the family tree of the BCG, are responsible for the largest fraction of the 'diffuse stellar component' that is observed in galaxy clusters. Semi-analytic models have only recently turned their attention to this component, and often model its formation only through tidal stripping [27, 14].

Gas stripping : Galaxies travelling through a dense intra-cluster medium suffer a strong ram-pressure stripping that can sweep cold gas out of the stellar disc [13]. Depending on the binding energy of the gas in the galaxy, the intra-cluster medium will either blow through the galaxy removing some of the diffuse interstellar medium, or will be forced to flow around the galaxy [6, 21]. Ram-pressure stripping is expected to be more important at the centre of massive systems because 

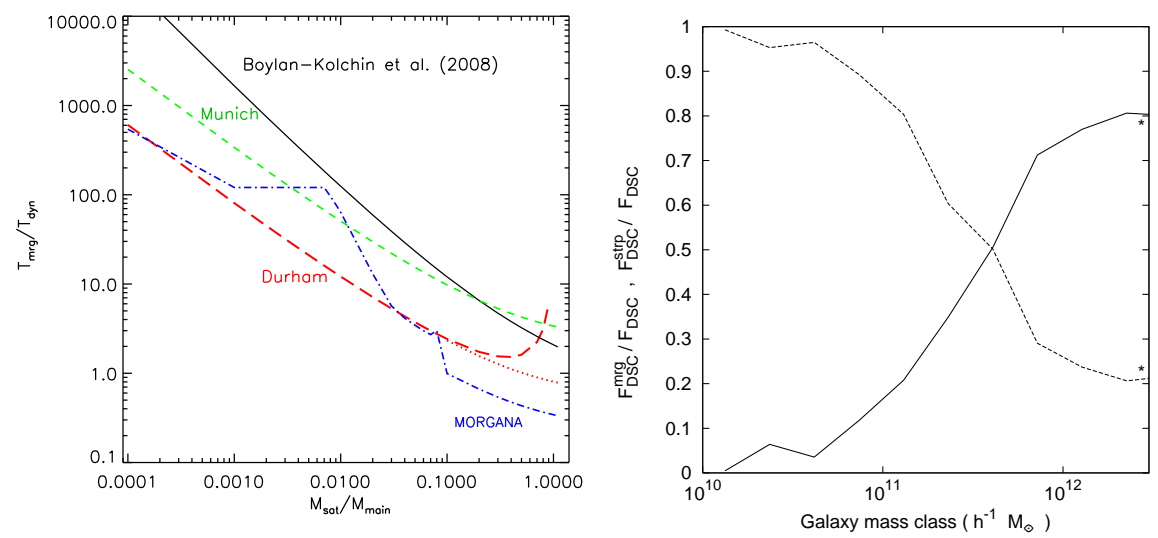

Fig. 1 Left: Merger times (in units of dynamical times) as a function of the baryonic mass ratio. Dashed, long-dashed and dot-dashed lines correspond to the standard assumptions adopted by the Munich, Durham and MORGANA models respectively. The thick solid line corresponds to the fitting formula provided by [2], for circular orbits. Right: The fraction of diffuse stars arising from mergers associated with the assembly history of the BCG (solid line), and with tidal stripping of stars from galaxies orbiting in the cluster potential (dashed line).

of the large relative velocities and higher densities of the intra-cluster medium. By considering the distribution and 'history of ram-pressure' experienced by galaxies in clusters, [4] estimated that strong episodes of ram-pressure are indeed predominant in the inner core of the clusters. They also showed, however, that virtually all cluster galaxies suffered weaker episodes of ram-pressure, suggesting that this physical process might have a significant role in shaping the observed properties of the entire cluster galaxy population. In addition, [4] found that ram-pressure fluctuates strongly so that episodes of strong ram-pressure alternate to episodes of weaker ram pressure, possibly allowing the gas reservoir to be replenished and intermittent episodes of star formation to occur.

While both numerical simulations and analytic studies show that ram-pressure stripping affects significantly the amount of gas in cluster galaxies, this physical process is usually not included in semi-analytic models of galaxy formation, with the exception of a couple of studies [23, 22]. These conclude that the inclusion of this physical processes causes only mild variations in galaxy colours and star formation rates (I will explain why this is the case below - see 'strangulation' section). It should be noted that these studies include ram-pressure stripping using the original analytic formulation proposed by [13]. Recent numerical work [25] has shown that this formulation often provides incorrect mass-loss rates. In addition, the simple models used so far do not consider the possibility that ram-pressure stripping could temporarily enhance star formation [29, 4].

Strangulation : Current theories of galaxy formation assume that, when a galaxy is accreted onto a larger structure, the gas supply can no longer be replenished by cooling that is suppressed by the removal of the hot gas halo associated with the 

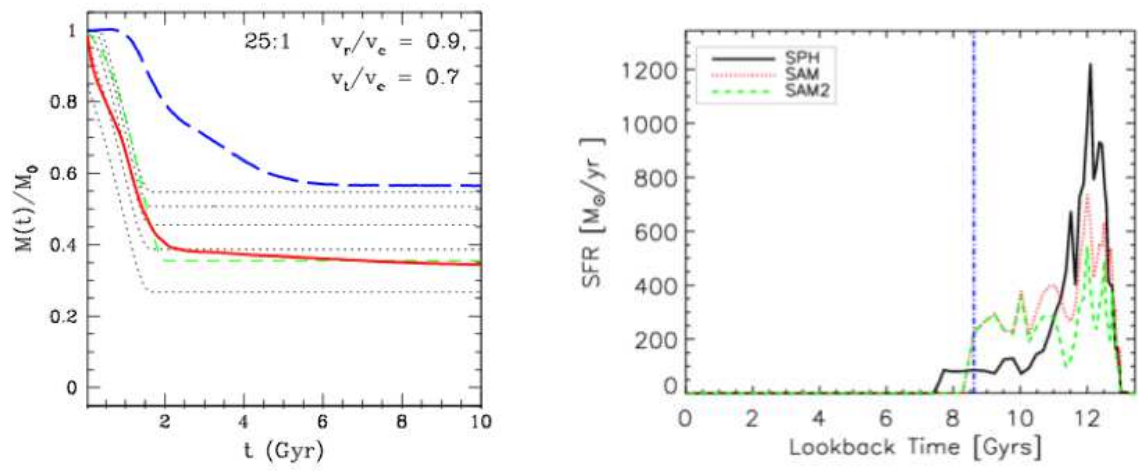

Fig. 2 Left: From [17]. The solid and dashed lines show the evolution of the bound mass of gas and dark matter in a simulation (see original paper for details) of a galaxy accreted onto a larger system. Right: From [26]. The star formation history of a galaxy residing in a cluster today. The vertical line indicates the time of accretion. The solid line is from a hydrodynamical simulations while the dashed and dotted lines are from a semi-analytic model (see original paper for details).

infalling galaxy [15]. This process is usually referred to as 'strangulation' (or 'starvation' or 'suffocation'). It is common to read in discussions related to these physical mechanisms, that strangulation is expected to affect the star formation of cluster galaxies on relatively long timescales, and therefore to cause a slow decline of the star formation activity. In semi-analytic models, however, this process is usually associated to a strong supernovae feedback, and is assumed to be instantaneous. As a consequence, galaxies that fall onto a larger system consume their cold gas rapidly, moving onto the red-sequence on very short time-scales (see e.g. [30]). This is also why ram-pressure stripping is not found to have a significant influence in these models: galaxies turn red and dead so quickly that ram-pressure does not have enough time to affect their colours or star formation rates.

Numerical simulations have recently pointed out that the stripping of the hot halo associated with infalling galaxies should not happen instantaneously. The left panel from Fig. 2 is reproduced from [17] and shows the evolution of the dark matter (solid line) and gas component (dashed line) associated with a galaxy placed on a realistic orbit through a cluster (see original paper for details). This particular simulation shows that the galaxy can retain $\sim 30$ per cent of its initial hot gas even $\sim 10 \mathrm{Gyr}$ after accretion. The right panel is reproduced from [27] and compares the star formation history of a cluster satellite galaxy from hydrodynamical simulations and semi-analytic models. [27] find that some cooling occurs on satellites and that this can last up to $\sim 1 \mathrm{Gyr}$ after accretion, but this seems to be important only for the most massive satellites. These simulations provide important (albeit still inconclusive) inputs for a revised treatment of this physical process in the framework of semi-analytic models of galaxy formation (see e.g [11, 31]).

Harassment : Galaxy harassment is a process that is not usually included in semi-analytic models of galaxy formation. The process has been discussed in early 

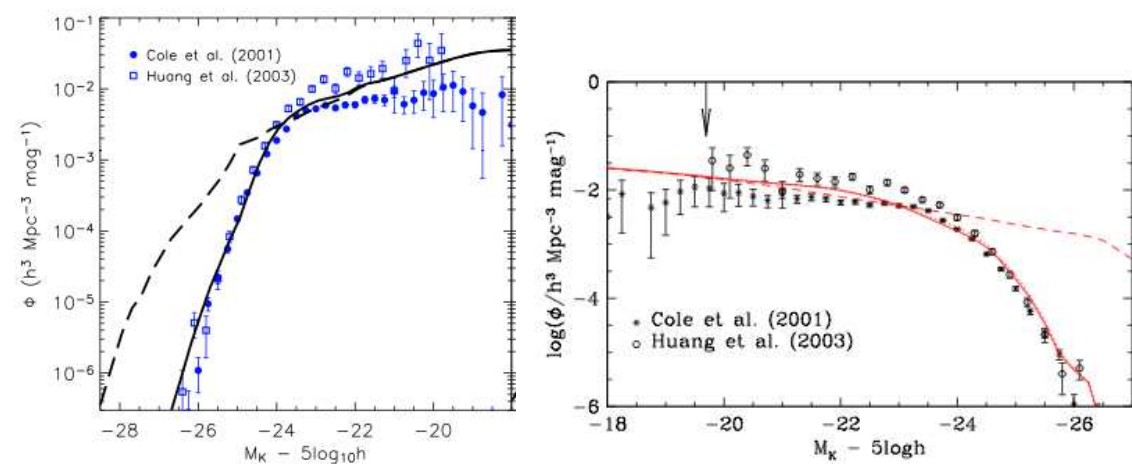

Fig. 3 Left: The predicted K-band luminosity function from two recently published semi-analytic models ([7] left panel and [3] right panel). In both panels, the solid and dashed lines show model predictions with and without AGN feedback, respectively.

work on the dynamical evolution of cluster galaxies [24], and has been explored in detail using numerical simulations [10, 19]. These have shown that repeated fast encounters, coupled with the effects of the global tidal field of the cluster, can drive a strong response in cluster galaxies. The efficiency of the process is, however, largely limited to low-luminosity hosts, due to their slowly rising rotation curves and their low-density cores. Therefore, it is believed that harassment might have an important role in the formation of dwarf ellipticals or in the destruction of low-surface brightness galaxies in clusters [16], but it is less able to explain the evolution of luminous cluster galaxies.

AGN heating: Since the milestone paper by [33], it has been realized that some physical process is needed to suppress cooling flows at the centre of relatively massive haloes. Early semi-analytic models introduced ad-hoc prescriptions to suppress cooling flows in haloes above a critical mass. Modern models have included more accurate and physically motivated prescriptions, and have confirmed that AGN heating is indeed important to reproduce the exponential cut-off at the bright end of the galaxy luminosity function, and the old stellar populations observed for massive galaxies [8]. Fig. 3] shows, for example, the predicted K-band luminosity function from two recently published semi-analytic models [3, 7] with and without AGN feedback (solid and dashed lines, respectively). The prescriptions adopted in these models are, however, necessarily very schematic and not well grounded in observation. A recent work by [12], in particular, has pointed out that the distributions of radio sources predicted by recent semi-analytic models is in disagreement with observational data, confirming that much work still needs to be done in order to understand exactly how and when AGN feedback is important. 


\section{What next?}

The above discussion highlights that there are several areas where our galaxy formation models could and should be improved. In particular:

- Different treatments of galaxy mergers lead to merger times that can differ up to one order of magnitude [9]. This has important consequences for the assembly history of the most massive galaxies, and for the evolution of the bright/massive end of the luminosity/mass function.

- Only recently, galaxy formation models have turned their attention to the formation of the diffuse stellar component. The increasing amount and quality of observational data on the intra-cluster light, and detailed comparisons with theoretical models, will provide important constraints on how this component forms and evolves as a function of cosmic time.

- Much recent work has focused on improving our treatment of gas stripping from satellite galaxies [14, 31]. These models, however, are not without problems: e.g. the model discussed in [14] still predicts a larger passive fraction among low-mass galaxies than is observed, and an excess of intermediate to low mass galaxies beyond $z \sim 0.5$. There results call for a deep revision of our modelling of the evolution of satellite galaxies.

- 'Radio-mode' AGN feedback represents an elegant solution to a number of longstanding problems related to the evolution of massive galaxies. Yet, our modelling of this physical process is still very schematic. In particular, the strong dependency on halo mass usually adopted in semi-analytic models might not be supported by observational data [12].

To conclude, I would like to remind that the importance of the physical processes discussed above has been investigated in detail using numerical simulations, at the typical velocity dispersions of galaxy clusters. Detailed studies at smaller scales (those of galaxy groups) are often lacking, even though groups likely represent the most common environment galaxies experience during their lifetimes.

The increasing amount and quality of the data being collected in these years are going to continuously challenge available and future models. This close link between theory and observations needs to be maintained (and strengthened) in order to improve our understanding of the processes driving galaxy formation and evolution as a function of the environment.

Acknowledgements I wish to thank A. Pasquali and I. Ferreras for the organization of a very lively and stimulating meeting. I acknowledge financial support from the European Research Council under the European Community's Seventh Framework Programme (FP7/2007-2013)/ERC grant agreement n. 202781. 


\section{References}

1. Barnes J. E., Hernquist L., 1996, ApJ, 471, 115

2. Boylan-Kolchin M., Ma C.-P., Quataert E., 2008, MNRAS, 383, 93

3. Bower R. G., Benson A. J., Malbon R., Helly J. C., Frenk C. S., Baugh C. M., Cole S., Lacey C. G., 2006, MNRAS, 370, 645

4. Brüggen M., De Lucia, G., 2008, MNRAS, 383, 1336

5. Cox T. J., Jonsson P., Somerville R. S., Primack J. R., Dekel A., 2008, MNRAS, 384, 386

6. Cowie L. L., Songaila, A., 1977, Nature, 266, 501

7. Croton D. J., Springel V., White S. D. M., De Lucia G., Frenk C. S., Gao L., Jenkins A., Kauffmann G., Navarro J. F., Yoshida N., 2006, MNRAS, 365, 11

8. De Lucia G., Springel V., White S. D. M., Croton D., Kauffmann G., 2006, MNRAS, 366, 499

9. De Lucia G., Boylan-Kolchin M., Benson A. J., Fontanot F., Monaco P., 2010, MNRAS, 406, 1533

10. Farouki R., Shapiro S. L., 1981, ApJ, 243, 32

11. Font A. S., Bower R. G., McCarthy I. G., Benson A. J., Frenk C. S., Helly J. C., Lacey C. G., Baugh C. M., Cole S., 2008, MNRAS, 389, 1619

12. Fontanot F., Pasquali A., De Lucia G., van den Bosch F. C., Somerville R. S., Kang X., 2010, MNRAS submitted, arXiv: 1006.5717

13. Gunn J. E., Gott J. R. I., 1972, ApJ, 176, 1

14. Guo Q., White S. D. M., Boylan-Kolchin M., De Lucia G., Kauffmann G., Lemson G., Li C., Springel V., Weinmann S., MNRAS in press, arXiv:1006.0106

15. Larson R. B., Tinsley B. M., Caldwell C. N., 1980, ApJ, 237, 692

16. Mastropietro C., Moore B., Mayer L., Debattista V. P., Piffaretti R., Stadel J., 2005, MNRAS, 364, 607

17. McCarthy I. G., Frenk C. S., Font A. S., Lacey C. G., Bower R. G., Mitchell N. L., Balogh M. L., Theuns T., 2008, MNRAS, 383, 593

18. Mihos J. C., 2004, in Clusters of galaxies: probes of cosmological structure and galaxy evolution, p.278, eds. J.S. Mulchaey, A. Dressler, A. Oemler, Cambridge University press

19. Moore B., Lake G., Katz N., 1998, ApJ, 495, 139

20. Murante G., Giovalli M., Gerhard O., Arnaboldi M., Borgani S., Dolag K., 2007, MNRAS, 377,2

21. Nulsen P. E. J. 1982, MNRAS, 198, 1007

22. Lanzoni B., Guiderdoni B., Mamon G. A., Devriendt J., Hatton S., 2005, MNRAS, 361, 369

23. Okamoto T., Nagashima M., 2003, ApJ, 587, 500

24. Richstone D. O., 1976, ApJ, 204, 642

25. Roediger E., Brüggen M., 2007, MNRAS, 380, 1399

26. Saro A., De Lucia G., Borgani S., Dolag K., 2010, MNRAS, 406, 729

27. Somerville R. S., Hopkins P. F., Cox T. J., Robertson B. E., Hernquist L., 2008, MNRAS, 391,481

28. Toomre A., Toomre J., 1972, ApJ, 178, 623

29. Vollmer B., Cayatte V., Balkowski C., Duschl W. J., 2001, ApJ, 561, 708

30. Wang L., Li C., Kauffmann G., De Lucia G., 2007, MNRAS, 377, 1419

31. Weinmann S. M., Kauffmann G., von der Linden A., De Lucia G., 2010, MNRAS, 406, 2249

32. White S. D. M., Rees M. J., 1978, MNRAS, 183, 341

33. White S. D. M., Frenk C. S., 1991, ApJ, 379, 52 